\title{
Inhaltsverzeichnis Jahrgang 1956
}

\section{Aufsätze}

Uupree, L.: Shamshir Ghar, a historic cave site in Kandahar province, Afghanistan. . . . . . . Junker, H.: Persepolis

Kraemer, J.: Zur Neuauflage von Brockelmanns Arabischer Grammatik.

Lanczkowski, G.: Zur Herkunit der Hyksos

Mayrhofer, M.: Altindische Nominalbildung. Zum neuen Band der "Altindischen Grammatik" .

otten, H.: Ein Text zum Neujahrsfest aus Boğazköy

\section{Besprechungen}

Aalto, P.: Altaistica, s. Wallin-Gedenkschrift - s. Schmidt, G

A hegg, L.: Neue Herren in Mittelost (W. Björk$\mathrm{man}$ )

Adam, A.: "Texte zum Manichäismus ausgewählt und hrsg. (A. Böhlig)

Alyangar, K. V. R.: Krtyakalpataru of Bhatta Laksmìdhara. VI (Fr. Weller) . . . . .

Albright, W. F.: Grundlagen und Entfaltung der ältesten Hochkulturen (W. Schubart)

- Die Religion Israels im Lichte der archäologischen Ausgrabungen. Ubers. mit Nachträgen v. F. Cornelius (O. EiBfeld $)$

1lt, A.: Die Herkunft der Hyksos in neuer Sicht (G. Lanczkowski)

[Anderson-Festschrift:] Beiträge zur vergleichenden Erzählforschung. Festschrift für Walter Anderson zum 70. Geburtstag, hrsg. v. K. Ranke (F. GeiBler)

Apted, M. R., s. Blackman, A.

Arsenjew, W.: Durch die Urwälder des Fernen Ostens (G. Köhler) . . . . . . . . . . .

Baehr, U.: Tafeln zur Behandlung chronologischer Probleme. I-III (F. Cornelius)

Bailey, H. W.: Indo-Scythian Studies $=$ Khotanese Texts II. (J. C. Tavadia $\dagger$ )

Balkan, K.: Kassitenstudien. I. Die Sprache der Kassiten. Aus dem Türk. übers. v. Fr. R. Kraus (R. Borger) .. . . . . . . .

Barnett, R. D., s. Dupont-Sommer, A.

Barthelemy, D., u. Milik, J. T.: Discoveries in the Judaean Desert. 1. Qumran Cave I. (O.E i B f el d t)

Bauer, Th.: Akkadische Lesestücke. I-III (H.-S. Schuster)

Baumgärtel, E. J.: The Cultures of Prehistoric Egypt. I. 2 nd rev. ed. (E. Otto) . . . . .

Bengtson, H., s. Weltatlas, Großer Historischer

Benl, 0.: Seami Motokiyo und der Geist des NóSchauspiels (M. R amming)

Bhagavadgita: Gesang des Erhabenen (W. Ruben)

Birkeland, H.: The Evildoers in the Book of Psalms (O. EiBfeldt)

Blackman, A. M., u. Apted, M. R.: The Rock Tombs of Meir. V u. VI. (H. Kees).

Blumhardt, J. F.: Catalogue of the Gujarati and Rajasthani Manuscripts in the India Office Library rev. and enlarged by A. Master (J. C. Tavadia $t$ )

Bodde, D., s. Wilson, $\dot{j} . \dot{A}$.

Böhl, F. M. Th. De Liagre: Opera Minora (E. Ebeling $\dagger$ )

- Tabula Cuneiformae. I. (H. Petschow).

Bomann, T.: Das hebräische Denken im Vergleich mit dem griechischen (H. Bardtke) . . . . .
Sossard, R.: Wege zur Selbstverwirklichung (W.

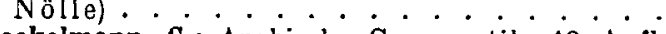

Brockelmann, C.: Arabische Grammatik. 13. Aufl. 293 - Osttürkische Grammatik der islamischen LitteratursprachenMittelasiens.Lig.2-6 (J.Németh) 444 Bruno, A.: Jesaja (F. Ma ass) . . . . . . . 235

389 - Die Bücher Genesis-Exodus (F. Ma ass) . . . 235

- Jeremia (F. Maass) . . . . . . . . . 235

5 - Die Psalmen (F. Ma ass) . . . . . . . . . . 235

101 - Die Bücher Samuel (F.Maass) . . . . . . . 237

- Die Bücher Könige (F. Maass) . . . . . . . 237

- Die Bücher Josua, Richter, Ruth (F. Maass) . 426

Buck, A. de: The Egyptian Coffin Texts. V. (H. Kees)

Buck, F. H.: Comparative Study of Postpositions in Mongolian Dialects and the Written Language (P. Ratchnevsky)... . . . . . . . .

Cantineau, J., s. Fück, J.

Cappeller, C.: Sanskrit-Wörterbuch nach den Petersburger Wörterbüchern bearb. (U. Schneider)

Cardaseia, G.: Les Archives des Murašû (G. Wallis)

Champdor, A.: Thèbes aux Cent Portes (G. Roeder)

Chéhadé, A.-K.: Ibn an-Nafîs et la Découverte de la Circulation Pulmonaire (A. Siggel) . . . . Cornelius, F.. s. Albright, W. F.

203 Crawford, Y. E.: Sumerian Economic Texts from the First Dynasty of Isin (K. Oberhuber) . . .

Crazzolara, J. P.: Zur Gesellschaft und Religion der Nueer (D. Westermann) . . . . . . . . .

Cuisinier, J.: La danse sacrée en Indochine et en Indonésie (A. A. Bake) . . . . . . . . . . Danby, H., s. Haimonides

Debrunner, A., s. Wackernagel, J

Denizeau, C., s. Fück, J.

495 Dentan, R. C.: The Idea of History in the Arcient Near East (O. EiBieldt).

Dieke, R., s. Ropers, H.

72 Douglas, W. 0.: Gärender Orient (W. Hinz) . . .

Dupont-Sommer, 1.: The Jewish Sect of Qumran and the Essenes. Transl. by R. D. Barnett (O. Ei Bfeldt) . . . . . . . . . . .

Dussaud, R.: La pénétration des Arabes en Syrie avant l'Islam (A. Dietrich) . . . . . .

Ebeling, E. $\dagger$ : Literarische Keilschrifttexte aus Assur hrsg. (J. Nouga yrol). . . . . . . . . . - Stiftungen und Vorschriften iür assyrische Tempel (R. Frankena) . . . . . . . • •

5 Eberhard, W.: Conquerors and Rulers (A. F. P. Hulsewé) The Record of a Pilorimage to China

Ennin's Diary. The Record of a Pilgrimage to China in Search of the Law. Transl. by E. O. Reischauer

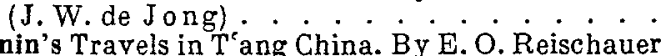

Ennin's Travels in T'ang China. By E. O. Reischauer (J.W. de Jong) . . . . . . . . . . . . .

Erdmann, K.: Arabische Schriftzeichen als Ornamente in der abendländischen Kunst des Mittelalters (F. Bajraktarević) - $\cdot \dot{\cdot} \cdot \cdot \cdot \cdot \cdot \cdot \cdot$

Erkes, E.: Neue Beiträge zur Geschichte des Choukönigs $\mathrm{Yu}$ (T. Pokora)

3 Erman, A. †: Kurzer Abriß der ägyptischen Grammatik. 4. Aufl. (W. Westendorf) . - - $\cdot$ -

- u. Grapow, H.: Wörterbuch der ägyptischen Sprache. Die Belegstellen. Bd. IV u. V (A.

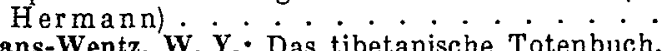

Evans-Wentz, W. $\mathbf{Y}$ : $\dot{D}$ as tibetanische Totenbuch, hrsg. u. übers. v. L. Göpfert-March. 5. Aufl.

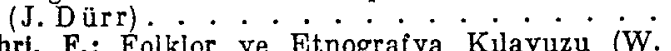
ahri, F.: Folkior ve Etnograíya Kilavuzu (W. B jörkman) . . . . . . . . . . . . . . . . 59

48 The Far East and South-East Asia (I. Kluge) . . 263 
Farhâdi, A bd-ul-Ghafûr: Le Persan parlé en Afghanistan (J. Cejpek u. J. R y p ka)

Farkas, J. v., s. Ural-altaische Jahrbücher

Farrukh, 0.: Two Arab Poets (W. Björ kman) . .

Frankfort, H.: The Birth of Civilization in the Near East (A. Moortgat)

Fronzaroli, P.: Leggenda di Aqhat (O. EiBfeldt)

Frye, R. N.: The History of Bukhara. Transl. by Narshakhī (W. Hinz)

Fuchs, W., v. Glasenapp, H., Gundert, W., Hansen, 0. Jaspers, K., Mensching, G., Paret, R., Schoeps, H. J.: Die großen nichtchristlichen Religionen unserer Zeit in Einzeldarstellungen (A. Sc himmel-Tar 1 )

Füek, J.: 'Arabíya. Trad. C. Denizeau. Ed. J. Cantineau (H. Giesecke)

Gemser, B.: Hebreeuse Spraakkuns (R. Meyer)

Glasenapp, H. F.: Buddhismus und Gottesidee (W. Ruben)

- Kant und die Religionen des Ostens (W. Ruben) Die Religionen der Menschheit (G. Mensching) - s. Fuchs, W.

Göpfert-March, L., s. Evans-Wentz, W. Y.

Goldin, J.: The Fathers According to Rabbi Nathan, ransl. from the Hebrew (R. Meyer)

Gonda, J.: Aspects of early Visṇuism (E. Frauwallner)

Gordon, C. H.: Smith College Tablets. 110 Cuneiform Texts selected from the College Collection (K. Oberhuber)

Grapow, H.: Von den medizinischen Texten (GrundriB der Medizin der Alten Ägypter II) (G. B. Gruber)

- Worte des Gedenkens an Adolf Erman anläßlich seines 100. Geburtstages (G. Roeder). s. Erman, A.

Greenberg, M.: The Hab/piru (O. EiBfeldt) .

Groenewegen-Frankfort, H. A.: Arrest and Movement ( $G$. Roeder)

Guenther, K., s. Schweinfurth

Güterbock, H. G., s. Wilson, J. A.

Gundert,W., s. Fuchs, W.

Gutersohn, H.: Indien (H. Losch)

Guyer, $S$, Grundlagen mitt ländischer Baukunst (J. K ollwitz)....

Hall, A. R., s. Singer, Ch.

Hambis, L., s. Polo, M.

al-Hamdānī: Al-Iklīl. I. Buch, hrsg. v. O. Löfgren. H. 1 (H. Mžik)

- Südarabisches Muštabih, hrsg. v. O. Löfgren (H. Mž i k)

Hansen, K. H.: Das iranische Königsbuch (j.R y p ka)

Hansen, 0., s. Fuchs, W.

[Han Yü:] The veritable Record of the T'ang Emperor Shun-Tsung. Han Yü's Shun-tsung shih-lu. Transl. by B. S. Solomon (T. Grim m)

Harder, E.: Kleine Arabische Sprachlehre. 5. Aufl. bearb. von R. Paret (A. Schimmel-Tarı).

Harrison, B.: South-East Asia (M. H. van der Val k)

Hartman, S.: Gayomart. Etude sur le syncretisme dans l'Ancien Iran (H. J unker) . . . . . . .

Hartmann, P.: Nominale Ausdrucksformen im wissenschaftlichen Sanskrit (W. Rau) .

Hauer, F. $\dagger$ : Handwörterbuch der Mandschusprache. Lfg. 1-3 (K. Grønbech)

Helck, W.: Untersuchungen zu den Beamtentiteln des ãgyptischen Alten Reiches (S. Sch ot t). .

Henninger, J.: Ist der sogenannte Nilus-Bericht eine brauchbare religionsgeschichtliche Quelle? (R. Hartmann)

- Uber Sternkunde und Sternkult in Nord- und Zentralarabien (A. Spitaler) . . . . . .

Hickmann, H.: Le Métier de Musicien au Temps des Pharaons (E. Brunner-Traut).....
Spalte

Hölseher, G. †: Geschichtsschreibung in Israel (A

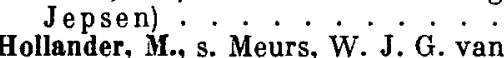

52 Hollister, J. N.: The Shi a of India (J. F üc k) . . . 245

Holma, H.: Georg August Wallin, s. Wallin-Gedenkschrift

136 Holmyard, F. J., s. Singer, Ch.

Hoppe, M.: Buddha. Seine Lehre und sein Weg (F. R. H am m) . . . . . . . . . . .

Hughes, G.-R.: Reliefs and Inscriptions at Karnak. III. ( $\mathrm{H}, \mathrm{K}$

Ingalls, D. H. H., s. Wilson, J. A.

Irak, Der (B. Spuler).

114 Irersen, E.: Canon and Proportions in Egyptian Art (H. Senk)

Jahrbuch des Museums für Völkerkunde zu Leipzig X u. XI. (S. Behrsing)

Jansky, H.: Lehrbuch der türkischen Siprache. 2. Aufl. (H. W. Duda) . . . . . . .

Jaspers, K., s. Fuchs, W.

Jean, Ch.-F. $\nmid$ : Dictionnaire des Inscriptions Sémitiques de l'Ouest. Lfg. 1 u. 2 (R. Me yer) . .

Jelowazki, I. P.: Malaya (H. D a mm) . . . .

Johnson, H.: Ein Viertel der Menschheit (J. Pr ù š e k).

Jyrkänkallio, P.: Zur Etymologie von russ, tolmą "Dolmetscher" und seiner türkischen Quelle, s. Wallin-Gedenkschrift

- Die sprachwissenschaftlichen Veröffentlichungen von Prof. Dr. M. Räsänen (A. v. Gabain)

ahrstedt, U.: Geschichte des griechisch-römischen Altertums (W. Schubart) . . . . . . .

Karnak-Nord. IV. (H. Kees).

317 Kavi, M. R.: Nātyasảstra with the commentary of Abhinavagupta. III. (W. Ruben) . . .

ees, H.: Das Priestertum im ägyptischenStaat vom Neuen Reich bis zur Spätzeit (H.Stock) . .

415 Keilschrifturkunden ans Boghazköi H. 36. Von H. Otten (E. Laroche)
Kielland, E. C.: Geometry in Egyptian Art (G. Roeder

Kirfel, W.: Das Purāna vom Weltgebäude (Bhuvanavinyāsa) (W. Rau) . . . . . . . . .

Kittel, G., s. Texte, Rabbinische

Klein, H., s. Maimonides

Köhler, O.: Geschichte der Erforschung der Nilotischen Sprachen (J. Lukas). . . . . . . . .

Kraemer, J., s. Nöldeke, Th.

Kraft, E. S.: Zum Dsungarenkrieg im 18. Jahrhundert (P. Ratchnevsky) .... . . . . . .

Krahe, H., s. Sommer-Festschrift

Kraus, F. R.: Wandel und Kontinuität in der sumerisch-babylonischen Kultur ( $\mathrm{J}$. Nougayrol) . s. Balkan, $\mathrm{K}$.

Kraus, H.-J.: Gottesdienst in Israel (Rud. Me yer)

- Die Königsherrschaft Gottes im Alten Testament (H. Bard tke) . . . . . . . . . . . .

Krishnamacharya, E., s. Viśveśvara Bhatta

Kühn, H.: Der Aufstieg der Menschheit (E. Str o mmenger) . . . . . . . . . . Kühnel, E., s. Kunst des Orients

61 Kuhl, C.: Die Entstehung des Alten Testaments (R. Meyer) . . . . . . . . . . . . . . . . Kunst des Orients, hrsg. v. E. Kühnel. H. 2 (K. Erdmann)................ 40

177 Kutscher, G., s. Sydow, E. v.

Lambton, A. K. S.: Persian Grammar (F. Bleiber) 156

W. A (A. Falkenstein).. . . . . . . . . . . .

146 - Legal and Economic Records from the Kingdom of Larsa (H. Petschow) . . . . . . .

145 Leipoldt, J., s. Till, W.

Littmann, E.: Arabische Märchen gesammelt u. übertr. (O. Spies)............. 
Löfgren, 0., s. al-Hamdān̄

Lommel, H.: Gedichte des Rig-Veda (W. Rau) .

Luiks, A. G.: Cathedra en Mensa (B. Spuler)

Mahābhärata, übers. v. V. Pisani (F. J. Meier) .

[Maimonides:] The Code of Maimonides X, transl. by H. Danby; XI. transl. by H. Klein (R. Meyer)

Marcais, Ph.: Textes Arabes de Djidjelli (W. Hoenerbach)

Master, A., s. Blumhardt, J. $\dot{F}$

Mende, T.: Südostasien zwischen zwei Welten (H. Franke)

Mendelsohn, I., s. Wilson, J. A.

Mensching, G., s. Fuchs, W.

Merlingen, W.: Das ,Vorgriechische" und die sprachwissenschaftlich - vorhistorischen Grundlagen (E. H of mann)

Meurs, W. J. G. van: Tibetan Temple Paintings, transl. by M. Hollander. 2 nd ed. (G. T ucri).

Milik, J. T., s. Barthélemy, D.

Milojciz, V., s. Weltatlas, Großer Historischer

Monteil, V.: Le persan contemporain. Textes et vo-

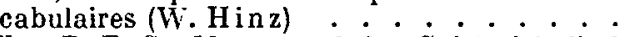

Müller, R. F. G.: Manas und der Geist altindischer Medizin (J.Filliozat) ..........

Nambiyar, M. R., s. Viśveśvara Bhattta

Narshakhī, s. Frye, R.

Neuronen, E. K.: La negacion $\vec{B}$ en el cancionero de Ibn Quzmān, s. Wallin-Gedenkschrift

Nobel, J.: Udrāyană, König von Roruka. I. (F. R. $\mathrm{H}$ a $\mathrm{mm}$ )

- dgl. II (F. R. H a m m)

Nöldeke, Th.: Belegwörterbuch zur klassischen ara bischen Sprache, bearb. u. hrsg. v. J. Kraemer. Lfg. 1 u. 2 (W. B jörkman).

North, R.: Sociology of the Biblical Jubilee (H Bardtke)

[Nyberg:] Donum natalicium H. S. Nyberg oblatum XXVIII mense dec. MCMLIV (W. B jörkman)

Ohm, T.: Die Religionen in Asien (G. Mensching)

olbricht, P.: Das Postwesen in China unter der Mongolenherrschaft im 13. u. 14. Jh. (E. Sch mit t + )

0'Shaughnessy, T.: The Derelopment of the Meaning of Spirit in the Koran (A. Schimmel-Tari)

Otten, H., s. Keilschrifturkunden aus Boghazköi

Otto, E.: Die biographischen Inschriften der ägyptischen Spätzeit (H. de Meulenaere)

Pallis, S. A.: Early Exploration in Mesopotamia (W. v. Soden)

Paret, R., s. Fuchs, W.

- s. Harder, E.

Parrot, A.: Golgotha et Saint-Sépulcre (O. Eiß-

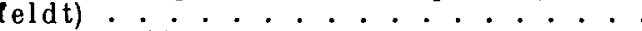

- Ninive et l'Ancien Testament (F.Schmidtke)

- Le Temple de Jérusalem (W. Rudolph) .

Pedersen, H.: Die gemeinindoeuropäischen und die vorindoeuropäischen VerschluBlaute (K. A m mer)

[Pedersen, J.:] Studia Orientalia. J. Pedersen Septuagenario (W. B jörkman) . . . . .

Pellat, Ch.: Le Milieu Basrien et la Formation de Găhiz (W. Caskel)

Piankoff, A., u. N. Rambova: The Tomb of Ramesses VI. (S. Schott) . . . . . . . . .

PIsani, V.: Storia delle Letterature antiche dell'India (W. Ruben)

- s. Mahābhảrata

Poebel, A.: The second Dynasty of Isin (A. Falkenstein)

Polo, M.: La Description du Monde, ed. L. Hambis (H. Plischke)

Poppe, N.: Grammar of Written Mongolian (E. Ha enisch)

Popper, W.: History of Egypt $1382-1469$ A. D Transl. from the Arabic Annals of Abu l-Mahâasin Ibn Taghrî Birdí. I u. II (W. B jörkman)
Spalte

Porada, E.: Seal Impressions of Nuzi (K. Schunck)

543 Prshewalski, N. M.: In das Land der wilden Kamele (G. Köhler) . . . . . . . .

Radhakrishnan, S.: Die Gemeinschaft des Geistes. Ubers. v. F. Thierfelder (G. Mensching) . • • Räsänen, M.: Beiträge zu den türkischen Runeninschriften, s. Wallin-Gedenkschrift

242 Rambova, N., s. Piankoff, A.

Ramstedt, G. J. †: Marginal Notes on Pashto Etymology, s. Wallin-Gedenkschrift

356 Ranke, K., s. Anderson-Festschrift

Reisehauer, E. 0., s. Ennin

Rengstori, K. H., s. Texte, Rabbinische

Renou, I.: Religions of Ancient India (W. Kirfel)

Reychman, J., s. Zajączkowski, A.

493 Ricciotti, G.: Geschichte Israels. I. (O. E i Bfeld t)

Riemschneider, M.: Die Welt der Hethiter (H. G. Güterbock) . . . . . . . . . . . . . .

Roemer, H. R.: Staatsschreiben der Timuridenzeit (V. Minorsky) . . . . . . . . . . .

Ropers, H.: Morgenländische Teppiche. 6. Aufl. bearb. v. R. Dieke (E. K ühnel) $\dot{\text {. }}$. * * * Rosenthal, F.: A History of Muslim Historiography (R. Hartmann)

Rostem, 0.R.: The Salvage of Philae (A.Herma n

Ruben, W.: Geschichte der indischen Philosophie (F. O. Schrader) . . . . . . . .

Rutten, M.: Les documents épigraphiques de Tchogha Zembil (W. Eilers) . - . . . . - *

Saarisalo, A.: Arabic Tradition and Topographical Research, s. Wallin-Gedenkschrift

Salmony, A.: Antler and Tongue (K. Finsterbusch) . A

Salonen, A.: Alte Substrat- und Kulturwörter im Arabischen, s. Wallin-Gedenkschrift

[Sauvaget:] Mémorial Jean Sauvaget. I. (R. Hart$\operatorname{man} n$ )

Schafer, E. H.: The Empire of Min (W. Franke) .

Schebesta, P.: Die Negrito Asiens. II, 1 (H. Plischke) $\therefore \cdot \dot{0} \therefore \dot{0} \dot{0} \cdot \dot{0}$

Schiffers, H.: Wilder Erdteil Afrika (H. Plischke)

Schmalenbach, W.: Die Kunst Afrikas (P. Ger-

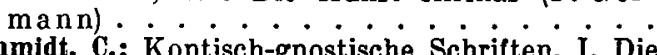

Schmidt, C.: Koptisch-gnostische Schriften. I. Di Pistis Sophia.

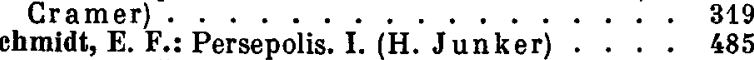

Sehmidt, G.: Uber Aufgaben und Methoden der Kaukasologie, hrsg. v. P. Aalto (G. Deeters) . . dgl. s. Wallin-Gedenkschrift

Sehmidt, W.: Gebräuche des Ehemannes bei Schwangerschaft und Geburt (W. Ruben) .

Sehoeps, H. J., s. Fuchs, W.

Schunck, K.-D.: Die Quellen des I. u. II. Makkabäerbuches (C. Kuhl)

[Sehweinfurth:] Georg Schweinfurth. Lebensbild eines Afrikaforschers, hrsg. von $K$. Guenther (W. Lang)

Shafer, R.: Ethnography of Ancient India (W.

*Kirfel) ...... . . . . . . . . .

Singer, Ch., Holmyard, E. J., u. Hall, A. R.: A History of Technology ed. (H. Quiring) :

Smith, F. H.: The Buddhist Way of Life (F. WelSmith, M.: The Șúfi Path of Love (A. SchimmelTar 1 )

Solomon, B. S., s. Han Yü

417 [Sommer, F.:] Corolla Linguistica. Festschrift F. Sommer zum 80. Geburtstag. Hrsg. v. H. Krahe (R. Werner) . . . . . Speiser, F. A., s. Wilson, J. A.

Spiegel, J.: Das Werden der altägyptischen Hochkultur (J. Leclant) . . . . . . . . . • Spuler, B.: Iran in früh-islamischer Zeit (I. H r bek 340 und J.Rypka)............. 
Stchoukine, I.: Les Peintures des Manuscrits Timúrides (E. Kühnel)

Steindorft, G.: Lehrbuch der koptischen Grammatik (E. Lüddeckens).

Stelninger, H.: Hauch- und Körperseele und der Dämon bei Kuan Yin-tze (C. Hentze)

Stler, F.: Das Buch Ijjob hebräisch und deutsch (G. $\mathrm{K} \cup \mathrm{hl}$ )

Storost, J.: Zur Aristoteles-Sage im Mittelalter (R. Hartman $n$ )

Stratil-Sauer, G.: Geographische Forschungen in Ostpersien. I. Die ostpersische Meridionalstraße (W. Hinz)

Sukenik, E. L.: The Dead Sea Scrolls of the Hebrew University ed. (O. Ei B feld t)

Sydow, E. v.: Afrikanische Plastik, hrsg. von G. Kutscher (P. Germann) . . . . . . . . .

Sylwan, Y.: Investigation of Silk from Edsen-gol and Lop-nor (W. Wegener)

Taghrî Birdî, s. Popper, W.

Texte, Rabbinische. 1. Reihe: Die Tosefta III, 1-3 VI, 5-6. 2. Reihe: Tannaitische Midraschim II, 1-9. Hrsg. v. G. Kittel u. K. H. Rengstorf (Rud. Me yer)

Thieme, P.: Die Heimat der indogermanischen Gemeinsprache (H. Krahe).

Thierfelder, F., s. Radhakrishnan, $\mathrm{S}$.

Tibetisch-mystische Lebensweisheit (J. D ürr)

Till, W. C.: Erbrechtliche Untersuchungen auf Grund der koptischen Urkunden (M. Krause)

- s. Schmidt, C.

- u. Leipoldt, J.: Der koptische Text der Kirchenordnung Hippolyts hrsg. u. übers. (M. Cramer)
Ural-altaische Jahrbücher. Fortsetz. der ,Ungar. Jahrbücher"6, hrsg. v. J.v. Farkas X XIV, 3-4

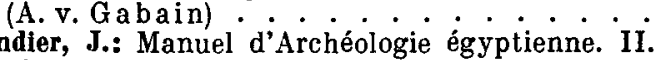

223 Vandier, J.: Manuel d'Archéologie égyptienne. II.

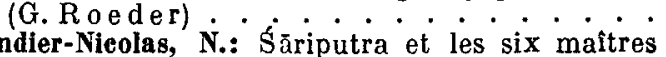

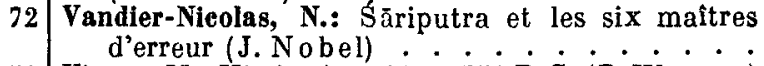

50 Vieyra, M.: Hittite Art $2300^{\circ}-750$ B. $\dot{\text { C. }}$ (R. $\dot{\text { Werner) }} 512$

Viśveśvara Bhatța: Madanamahārṇava, ed. by E. Krishnamacharya and M. R. Nambiyar (H. I. Ko hish

Wackernagel, J.: Altindische Grammatik. II, $\dot{2}: \dot{\text { Die }}$ Nominalsuffixe. Von A. Debrunner (M. Ma y rhofer) ................ . Wallin-Gedenkschrift:] In memoriam saecularem Georg August Wallin 1811-1852 (E.Lit t ma n n)

179 Weltatlas, Großer Historischer. I. 2. Aufl. Erläut. zu Teil I. von H. Bengtson u. V. Milojcić (O. Eißfeld t)

Widengren, G.: Sakrales Königtum im Alten Testament und im Judentum (F.Mass) . . . . .

Wiens, H. J.: China's March toward the Tropics (W. Eichhorn) . . . . . . . . . . . . .

336 Wilson, J. A., Speiser, E. A., Güterbock, H. G., Mendelsohn, I., Ingalis, D. H. H., u. Bodde, D.: Authority and Law in the Ancient Orient (O. EiBfeldt).

273 Wolf, W.: Die Welt der Ägypter (E. Otto) . . . .

Woolley, L.: Ein vergessenes Königreich (H. Ot te n) Zająezkowski, A., u. Reychman, J.: Zarys Diplomatyki Osmańsko-Tureckiej (L. Fekete) . . .

Zimmerli, W.: Erkenntnis Gottes nach dem Buche

33 Ezechiel (C. Ku hl) . . . . . . . . . 334

\section{Verzeichnis der Mitarbeiter}

Spalte

A mmer, $K$.

Bajraktarevic, F. $\cdot \cdot \cdot \cdot 341$

Bake, A. A. . . . . 357

Bardtke, H. . . . 48. 139. 239

Behrsing, S.

19. $\dot{59} \cdot 22$

59. 105 .

152, 338. 340. 442

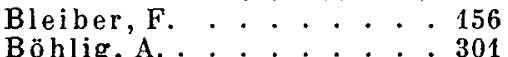

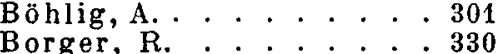

Brunner-Traut, E. . . 506

Caskel,W. ....... . 149

Cejpek,J........ . . 446

Cornelius, F. . . . . . . 399

Cramer, M. . . . . . 33. 319

Damm, H. . . . . . . . . 456

Deeters, G. . . . . . . 353

Dietrich,A. . 373. 374. 431. 557

Duda, H. W. . . . . . . . 154

Dürr, J. . . . . . . 273. 365

Dupree, L.. . . . . . . 293

Ebeling, E. $t$. . . . . . 35

Edel, E.... . . . . . 467

Eichhorn, W. . . . . . . . 268

Eilers, W........ . 45

Eibfeldt, $O$. 36. 55. 89. 136. 241. 279. 280. 337. 400. 415. 425 . 427. 488. 522. 523

Erdmann, K. 402 Falkenstein, A. . . . 328. 417 Fekete, L. . . . . . . . 539

Filliozat, J....... 261

Finsterbusch, K. . . . . 361

Fleischhammer, M. . . . . 282

Franke, H. ..... . 356

Franke,W. 552

Frankena, R. . . . . . 133
Spalte Spalte

Frauwallner, E. . . . . . 258 Laroche, E. . . . . . . . . 420

Fück, J........ . 245. 283 Leclant, J. . . . . . . . . 118

Gabain, A.v. . . . . 443. 537 Leipoldt, J. . . . . . . 185

GeiBler,F. . . . . . . 495 Littmann, E. . . . . . . 15

Germann, P..... . 179.372 Losch, H. . . . . . 256.355

Giesecke,H.H. . . . 90.531 Lüddeckens, E. . . . . . 223

Grimm, T. . . . . . 4 460 Lukas, J. . . . . . . 465

Grønbech, K. . . . . . 177 Maass, F. . 141. 235. 237. 426

Gruber, G. B. . . . . . . 317

Güterbock, H.-G. . . . . 513

Haenisch, E. . . . . . 172

$\mathrm{H} \mathrm{a} \mathrm{m} \mathrm{m,} \mathrm{F.} \mathrm{R.} \mathrm{.} \mathrm{.} \mathrm{.} \mathrm{72.} \mathrm{168.} 550$ Hartmann, R. 146. 401. 433. 531

Hentze, C. . Hinz, W... . 60. 159. 213. 352 Hoenerbach,W. . . . 242 H ofm ann, E. . . . . . 493 Hrbek, I. . . . . . . . . 248 Hulsewé, A. F. P. . . . . 170 Irmscher, J. 184. 189.281. 475.560 Jensen, H. . . . . . 185. 278 Jepsen, A. . . . . . . . 137 Jong, J.W. de...... . . 461 Junker, H. . . . . . 61, 485 Kees, H. 23. 125. 219. 409.474. 557 Kirfel, W. . . . . . . 63. 160 Kluge, I. . . . . . . 263.278 Köhler, G. . . . . . . 72. 264 Kollwitz, J. . . . . . . . 208 Kraemer,J. . . . . . . . 197 Krahe, H. . . . . . . . 205 Krause, M. . . . . . . 323 Kühnel, E. . . . . . 254. 408 $\mathrm{Kuhl}, \mathrm{G}$. . . . . . 50. 334. 524 Lanczkowski, G. . . . 389 Lang, W. . . . . . . . 275
Mayrhofer, M. . . . 5

Meier,F.J. . . . . . . . 164

Mensching, G. . 112. 281. 298. 299

Meulenaere, H. de . . . . 30

Meyer, Rud. 234. 332. 336. 424. 429. 466 . 525. 527

Minorsky, V. . . . . . . 348 Moortgat, A. . . . . . . . 490 Mžik, H. . . . 56. 57

Németh, J. . . . . . . . 444

Nobel, J. . . . . . . . . 358

Nölle,W. . . . . . . . . . 115

Nougayrol, J. . . . . 38. 511

Oberhuber, K. . . . 224. 231

Otto,E...... . 307. 504

Paret, R. . . . . 284. 373

Petschow, H. . . . . . 226

Plischke, H. . 262. 303. 371

Pokora, T. . . . . . . 77

Prúšek, J. . . 363

Quiring, H. . . . . . . . . 109

Ratchnevsky, P. . . 270. 456

$\mathrm{Rau}, \mathrm{W}$...... . 68. 450. 543

Roeder, G. 26. 304. 315. 504. 509

Ruben, W. . 64. 164. 260. 393 .

Rudolph,W. . . . . . . 144
Otten, H. . . . 93. 101. 232

Ramming, M. . . . . . . . 554 


\begin{tabular}{|c|c|c|c|c|c|}
\hline & $s \mathrm{~s}$ & & & Spa & Spa \\
\hline$\dot{a r i}, \dot{A}$ & 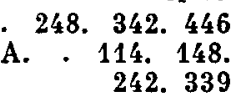 & $\begin{array}{l}\text { ter, H.-S. } \\
\text { H. } \cdot \text { : }: \\
\text { A. }: \text { : }\end{array}$ & & $\begin{array}{l}. \quad .128 \\
. \quad . \quad 215 \\
. \quad . \quad 440\end{array}$ & $\begin{array}{l}\text { Tucci, G. . } \\
\text { Valk, M. H. van der: } \\
\text { Wallis, G. . }\end{array}$ \\
\hline $\begin{array}{l}\text { Schmidtke, F. } \\
\text { Schmitt, E. } \dagger \text {. } \\
\text { Schneider, U. } \\
\text { Schott, S. } \\
\text { Schrader, F. } \\
\text { Schubart, W. } \\
\text { Schunck, K. }\end{array}$ & 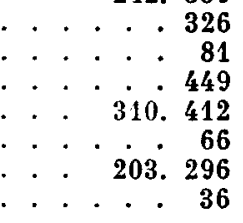 & $\begin{array}{l}\text { W. Frhr. v. } \\
\text { o. A. : } \\
\text { r, B. : : } \\
\text { imenger, E. } \\
\text { ia, J.C. } \dagger \text {. }\end{array}$ & & 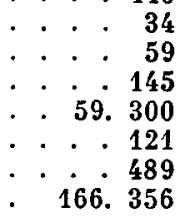 & 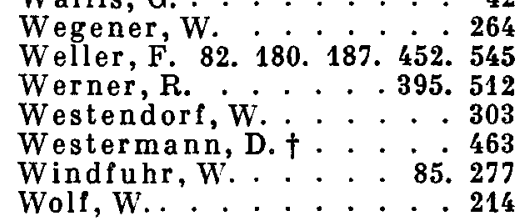 \\
\hline
\end{tabular}

Zeitschriftenschau: Acta Orientalia 373 - Al-Mašriq 373. 557 - Annales du Service des Antiquités 557 Archiv Orientální 374 - The Biblical Archaeologist 466 - Bibliotheca Orientalis 467 - Bulletin de l'institut français d'archéologie orientale $474-$ Byzantinische Zeitschrift 475. 560 - East and West $180-$ Harvard Journal of Asiatic Studies 82 - The Jewish Quarterly Review 85.277 - Journal of the American Oriental Society 89 - Journal Asiatique 90 - Journal of Cuneiform Studies 9.9 - The Journal of Hellenic
Studies 184 - Language 185.278 - Le Muséon $185-$ Nachrichten der Gesellschaft für Natur- und Völkerkunde Ostasiens Hamburg 278 - Oriens Extremus 187 - Palestine Exploration Quarterly 279 - Revue Biblique 280 - Revue des études byzantines 189.281 - Revue de l'histoire des Religions 281 - Studies in Bibliography and Booklore 282 - Wiener Zeitschrift für die Kunde des Morgenlandes 283 - Zeitschrift der Deutschen Morgenländischen Gesellschaft 284

Die , Orientalistische Literaturzeitnng“ erscheint im Akademie-Verlag, Berlin W 8, MohrenstraBe 39. Lizenzausgabe des J. O. Hinrichs Verlages, Leipzig O1, ScherlstraBe 2. Veröffentlicht unter der Lizenznummer 1205 desAmtes fürLuteratur und Verlagswesen der Deutschen Demokratischen Republik. Verantwortlicher Herausgeber Prot. D. Dr. Richard Hartmana, Berlin W 8, Unter den Linden 8. Jăhrlich 12 Hefte, Bezugspreis halbjährlich DM 30.-

Satz und Druck: Tribūne Druckerei III Leipzig III/18/36. Printed in Germany. Bestell- und Verlagsnummer: 1024/51 